\title{
AVALIAÇÃO DO ESTADO NUTRICIONAL DO FEIJOEIRO (Phaseolus vulgaris L.) CULTIVADO EM SOLUÇÃO NUTRITIVA, COM VARIAÇÃO NO FORNECIMENTO DE N, P e $\mathrm{K}$
}

\author{
R. CAMACHO'; A.M. CALVACHE'; N. FALCÃO'; F. FERNANDEZ"; J.A.M. DEMATTÉ; \\ E. MALAVOLTA ${ }^{s}$
}

'Universidad Rómulo Gallegos, "El Castrero", San Juan de los Morros, Guárico, Venezuela. Bolsista do Programa CONICIT-BID.

'Universidad Central del Ecuador - C.P. 2520, Quito - Ecuador. Bolsista da CAPES.

'Instituto Nacional de Pesquisa da Amazónia-INPAMCT, C.P. 478, CEP: 69083-000, Manaus,AM.

Departamento de Ciencia do Solo - ESALQ/USP, C.P. 9, CEP: 13418-900, Piracicaba,SP.

${ }^{3}$ Centro de Energia Nuclear na AgriculturaNSP, C.P. 96, CEP: 13400-970, Piracicaba,SP.

RESUMO: O feijoeiro (Phaseolus vulgaris L. cv. Carioca) foi cultivado em soluçăo nutrittva na presença das seguintes concentraç̃es de N, P e K, respectivamente em milimoles por litro: 16-2-6; 4-2-6; 1,6-2-6; 16-0,5-6; 16-0-6; 16-23; 16-2-0,6. Os outros macronutrientes e os micronutrientes foram fornecidos em doses iguais a todas as plantas. $O$ estado nutricional foi avaliado através das seguintes técnicas: diagnose follar (determinaçäo dos teores totais de $\mathbf{N}, \mathbf{P}$ e Ke da concentração de nitrato e fosfato solúvel), atividade da redutase do nitrato (RNO,). Todos os métodos utilizados refletiram bem o estado nutricional do feijoeiro, exceto para $P$, sendo que para a atividade da $\mathbf{R N O}_{3}$, os teores totais de $\mathrm{N}$ e $\mathrm{K}$ e de $\mathrm{N}$-nitrato foram os que apresentaram a melhor correlaçao positiva com a produçăo das vagens.

Descritores: feijoeiro, concentraç̄o de nutrientes, avaliaçāo do estado nutricional, redutase do nitrato

\section{EVALUATION OF THE NUTRITIONAL STATUS OF THE BEAN PLANT (Phaseolus vulgaris L.) GROWN IN NUTRIENT SOLUTION UNDER VARYING LEVELS OF N, P AND K}

\begin{abstract}
Bean plants (Phaseolus vulgaris L., cv. Carioca) were grown in nutrient solution in the presence of the following concentrations of $N, P$ and $K$, respectively, in milimoles per liter: 16-2-6; 4-2-6; 1,6-2-6; 16-0,5-6; 16-0-6; 16-2-3 and 16-2-0,6. The remaining macro and all micronut rients were supplied at the same rates to all plants. The nutritional status was assessed by the following techniques: foliar dlagnosis (determination of total $N, P$ and $K$ and of the nitrate- $\mathrm{N}$ and soluble phosphate concentration) and nitrate reductase activity (RNO, ). All methods proved to be suitable, except in the case of $P$. Higher correlations with bean production, however, were obtaind for total concentration of $\mathrm{N}$ and $\mathrm{K}$, nitrate- $\mathrm{N}$, and $\mathrm{RNO}_{3}$ activity.
\end{abstract}

Key Words: common bean, nitrate reductase, nutrient concentrations, evaluation of the nutritional status

\section{INTRODUÇÃO}

Muitos métodos têm sido desenvolvidos para avaliar o estado nutricional das plantas. Uma dessas técnicas em que se analisam determinadas folhas em períodos definidos de crescimento da planta, $\boldsymbol{c}$ a diagnose foliar, que consiste em analisar o solo usando a planta como solução extratora (MALAVOLTA et al., 1989).

A redutase do nitrato, enzima induzida por molibdênio e pelo substrato, tem sido utilizada para avaliar o estado nutricional com respeito ao $\mathrm{N}$ (BAR-AKIVA \& STERBAUM, 1965; BAR-AKIVA et al., 1970). E que existe, dentro de limites, correlação positiva entre suprimento de nitrogênio, atividade da enzima e produção.

O presente trabalho teve como objetivos avaliar o estado nutricional do feijoeiro (Phaseolus vulgaris L. cv. Carioca), cultivado em solução nutritiva, através da diagnose foliar e de um teste bioquímico para $\mathbf{N}$ (redutase do nitrato).

\section{MATERIAL E METODOS}

O feijoeiro (Phaseolus vulgaris L.) cultivar carioca foi cultivado em solução de 
JOHNSON et al. (1957) modificada por MALAVOLTA et al. (1993), de modo a fornecer 3 níveis de $\mathrm{N}, 3$ de $\mathrm{P}$ e 3 de $\mathrm{K}$. Os tratamentos foram os seguintes: (1) 16-2-6; (2) 4-2-6; (3) 1,6-2-6; (4) $16-0,5-6$; (5) $16-0-6$; (6) 16-2-3; (7) 16-2-0,6. Os três números se referem a milimoles respectivamente de $N$, P e K. As soluçōes eram constantemente arejadas e renovadas a cada 15 dias.

Os métodos de avaliação do estado nutricional usados foram: 1) diagnose foliar - teores solúveis de nitrato e fosfato - (adaptado de ULRICH, 1948) e teores totais de nitrogenio e fósforo (MALAVOLTA et al., 1989). 2) teste bioquímico para $\mathbf{N}$ - determinação da atividade da enzima redutase do nitrato - segundo procedimento descrito por MALAVOLTA et al. (1993).

Os dados foram submetidos à análise estatística (variância e regressão).

\section{RESULTADOS E DISCUSSÃO}

Crescimento e Produção: A maior produção de matéria seca total e nas vagens foram observadas no tratamento em que se forneceram os níveis mais altos de N, P e K. O menor rendimento ocorreu no tratamento com omissão de fósforo e com a menor dose de nitrogênio (TABELA 1). Observa-se que a produção foi mais limitada por $P$ e $N$ que por $K, 0$ que está de acordo com os resultados obtidos por MALAVOLTA et al. (1980). Percebe-se que o K, foi o nutriente que menos limitou a produção de vagens. $O$ potássio, na maioria das culturas, é um elemento tipicamente vegetativo, isto $\epsilon$, acumulase nas raízes, caules e folhas, aparecendo em teores relativamente baixos nos órgãos reprodutivos. Além disso, a renovação perí́dica da solução e mais a facilidade de redistribuição do $\mathrm{K}$ na planta, devem ter contribuído para o observado.

Existe uma alta correlação entre produção de matéria seca das vagens e dose de $\mathrm{N}$, $P$ e K, apresentando coeficientes de: 0,96; 0,99 e 0,98 , respectivamente.

Diagnose foliar: Na TABELA 2 encontram-se os conteúdos solúveis e totais de elementos nas folhas. Foram observadas diferenças altamente significativas entre a composição das plantas do tratamento 1 e as das plantas com deficiências de $\mathrm{N}, \mathrm{P}$ e K, demonstrando a possibilidade do uso destas técnicas para a avaliação do estado nutricional do feijoeiro. Os valores de $P$ (solúvel e total) foram relativamente mais altos no tratamento com ausência de $P$ que no tratamento 0,5 milimol $P$, devido possivelmente ao efeito da diluição(MALAVOLTA et al., 1989).

$O$ teor de $\mathbf{N}$ nas folhas das plantas do tratamento 1 pode ser considerado como adequado (COBRA NETO et al., 1971). Nos tratamentos com menor teor de $\mathrm{N}$, os teores seriam considerados como deficientes. Por outro lado para MALAVOLTA et al. (1989), os teores de $\mathrm{N}$ nos 3 tratamentos seriam deficientes; 0 teor de $P$ nas folhas das plantas do tratamento 1 seria considerado como alto;

TABELA 1 - Produção de matéria seca (g/vaso) em função dos tratamentos.

Tratamento

Matéria seca

\begin{tabular}{|c|c|c|c|c|c|c|}
\hline $\mathrm{N}^{\mathbf{0}}$ & $\mathbf{N} \mathbf{P} \mathbf{K}$ & Raiz & Ramos & Folhas & Vagens & Total \\
\hline & $\mathrm{mM}^{\mathbf{1}^{-1}}$ & & & g/vaso & & \\
\hline 1 & $16-2-6$ & $3,05 a *$ & 7,9ab & $13,26 \mathrm{ab}$ & $14,03 a$ & $38,23 a$ \\
\hline 2 & $4-2-6$ & $2,82 \mathrm{a}$ & $5,24 b c$ & $6,22 b c$ & $6,45 b c d$ & $20,73 c$ \\
\hline 3 & $1,6-2-6$ & $2,44 a$ & $2,26 \mathrm{~cd}$ & $3,18 \mathrm{~cd}$ & $2,79 \mathrm{de}$ & $10,56 d$ \\
\hline 4 & $16-0,5-6$ & $3,03 a$ & $10,57 \mathrm{a}$ & $11,45 \mathrm{a}$ & 5,22 cde & $30,26 \mathrm{~b}$ \\
\hline 5 & $16-0-6$ & $1,33 a$ & $1,17 d$ & $1,90 d$ & $2,12 \mathrm{e}$ & $6,51 d$ \\
\hline 6 & $16-2-3$ & $2,53 a$ & $8,66 \mathrm{ab}$ & $12,98 \mathrm{ab}$ & $9,91 b$ & $34,07 b$ \\
\hline 7 & $16-2-0,6$ & $2,21 a$ & $5,92 \mathrm{~b}$ & $8,14 b$ & $7,41 b c$ & $23,66 \mathrm{c}$ \\
\hline
\end{tabular}

* Valores na mesma coluna acompanhados com a mesma letra, não diferem entre si pelo teste de Tukey, a $5 \%$ de probabilidade. 
TABELA 2 -Teores de $\mathrm{N}-\mathrm{NO}_{3}$ e $\mathrm{P}_{2} \mathrm{H}_{2} \mathrm{PO}$ e totais de $\mathrm{N}, \mathrm{P}$ e K em folhas de feijoeiro, em função dos tratamentos.

\begin{tabular}{|c|c|c|c|c|c|c|}
\hline \multicolumn{2}{|c|}{ Tratamento } & $\mathrm{N}-\mathrm{NO}_{3}$ & $\mathbf{N}$ & $\mathrm{P}_{-} \mathrm{H}_{2} \mathrm{PO}_{4}$ & $\mathbf{P}$ & $\mathbf{K}$ \\
\hline & $\mathrm{mM} \mathrm{1}^{-1}$ & $\mathrm{mg} / \mathrm{kg}$ & $\mathbf{g} / \mathbf{k g}$ & $\mathrm{mg} / \mathrm{kg}$ & g/kg & $\mathrm{g} / \mathrm{kg}$ \\
\hline 1 & $16-2-6$ & $1035 \mathrm{a}^{*}$ & $25 a$ & $4.748 a$ & $6 a$ & $25 \mathrm{a}$ \\
\hline 2 & $4-2-6$ & $411 b$ & $17 a b$ & & & \\
\hline 3 & $1,6-2-6$ & $301 \mathrm{~b}$ & $14 \mathrm{~b}$ & & & \\
\hline 4 & $16-0,5-6$ & & & $1.006 \mathrm{c}$ & $1 \mathrm{c}$ & \\
\hline 5 & $16-0-6$ & & & $2.285 b$ & $3 \mathrm{~b}$ & \\
\hline 6 & $16-2-3$ & & & & & $16 \mathrm{~b}$ \\
\hline 7 & $16-2-0,6$ & & & & & $2 c$ \\
\hline
\end{tabular}

* Valores na mesma coluna acompanhados com a mesma letra não diferem entre si pelo Teste de Tukey, a $5 \%$ de probabilidade.

os teores observados nas plantas deficientes em $\mathbf{P}$ seriam baixos. No caso do $K$ o valor obtido no tratamento com $0,6 \mathrm{mM}$, seria deficiente para COBRA NETO et al. (1971).

Testes bioquímicos: A Figura 1 mostra o efeito da concentração do substrato sobre a atividade da $\mathrm{RNO}_{3}$. A produção de $\mathrm{N}-\mathrm{NO}_{2}^{-}$aumenta rapidamente nas menores concentrações de nitrogênio, tendendo a ficar constante a partir de $16 \mathrm{mM}$ de $\mathrm{N}$ na solução nutritiva. A partir dessa concentração, a enzima é considerada saturada com o seu substrato, sendo a atividade independente da concentração de nitrogênio exógeno. A equação de regressão para atividade da $\mathrm{RNO}_{3}$ (Y) em função da dose de nitrogênio $(X)$ encontrada foi $Y=7,8132+10,6647 X-0,51272 X^{2}$, com $r^{2}=0,95$.

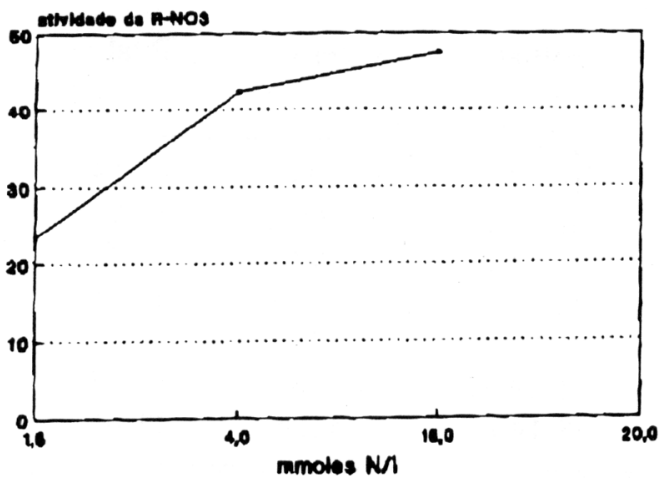

Figura 1 -Efeito da concentração do substrato (milimoles $\mathrm{N} / \mathrm{l}$ ), sobre a atividade da redutase do nitrato (ig $\mathrm{N}-\mathrm{NO}_{2}^{-} \cdot \mathrm{g}^{-1} \mathrm{mf} \cdot \mathrm{h}^{1}$ ).
Avaliação do estado nutricional: Para verificar a eficiência dos métodos de avaliação do estado nutricional, fizeram-se correlações entre a produção de matéria seca nas vagens (Y) e: teor de $\mathbf{N}$ total $\left(X_{1}\right)$, teor de $\mathrm{N}-\mathrm{NO}_{3}^{-}\left(\mathrm{X}_{2}\right)$, atividade da redutase do nitrato $\left(x_{3}\right)$, teor de $P$ total $\left(X_{4}\right)$, teor de $\mathrm{H}_{2} \mathrm{PO}_{4}$ $\left(X_{3}\right)$ e teor de $K$ total $\left(X_{6}\right)$.

Na TABELA 3 verifica-se que a diagnose foliar (para nitrogênio total e solúvel e potássio total) foi o melhor método para avaliar o estado nutricional do feijoeiro, devido a apresentar coeficientes de determinação mais altos.

TABELA 3 -Coeficientes de determinação $\left(r^{2}\right)$ entre a produção de matéria seca nas vagens (MS ) e a diagnose foliar e atividade da redutase do nitrato.

\begin{tabular}{|c|c|}
\hline Variavel & $r^{2}$ \\
\hline $\mathbf{M S}_{\checkmark} \times \mathbf{N}$ total & 0,997 \\
\hline $\mathrm{MS}_{v} \times \mathrm{N}-\mathrm{NO}_{3}$ & 0,967 \\
\hline $\mathrm{MS}_{4} \times \mathrm{R}-\mathrm{NO}_{3}$ & 0,766 \\
\hline $\mathbf{M S}_{\mathrm{v}} \times \mathbf{P}$ total & 0,622 \\
\hline $\mathrm{MS}_{v} \times \mathrm{P}-\mathrm{H}_{2} \mathrm{PO}_{4}^{-}$ & 0,699 \\
\hline $\mathbf{M S}_{\mathrm{v}} \times \mathbf{K}$ total & 0,94 \\
\hline
\end{tabular}

\section{CONCLUSÕES}

1) As plantas deficientes em $N, P$ e $K$ apresentaram baixa produção de matéria seca nas vagens e planta toda. 
2) Os teores solúveis de $\mathrm{N}$ e totais de $\mathrm{N}$ e $\mathrm{K}$, apresentaram a melhor correlação positiva com a produção de vagens, mostrando que a diagnose

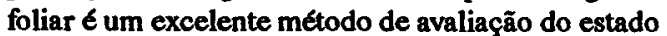
nutricional do feijoeiro.

3) A determinação de atividade da redutase do nitrato foliar mostrou-se capaz de descrever a influência do nível de $\mathrm{N}$ no substrato com a produção.

\section{AGRADECIMENTOS}

Participaram da elaboração e execução deste trabalho: A.A.C. Salviano, A.C.C. Bernardi, A.L.M. Menck, E.C. Brasil, E.P. Neto, F. Broetto, F.C. Oliveira, H.S. Pereira, J.A. Scarpare Filho, J.A.A. Silva, J.S. Holanda, M.C.S. Carvalho, M.R.L. Rodrigues, R.R. Beltran, R.J.M. Hernandez, S.R. Penteado, U. Gabe, V.M. Silva, I.M.T. Piza, M.Y. Nishimura e A.A. Gallego.

\section{REFERÊNCIAS BIBLIOGRÁFICAS}

BAR-AKIVA, A.; STERBAUM, J. Possible use of the nitrate reductase activity of leaves as a measure of the nitrogen requirement of Citrus trees. Plant and Cell Physiology, v.6, p.575-577, 1965.

BAR-AKIVA, A.; SAGIV, J.; LESHEM, J. Nitratereductase activity as an indicator for assesing the nitrogen requirement of grass crops. Journal of Science and Food Agriculture, v.21, p.405-407, 1970.
COBRA NETTO, A.; ACCORSI, W.R.; MALAVOLTA, E. Estudos sobre a nutrição mineral do feijoeiro (Phaseolus vulgaris L. var. roxinho). Anais da Escola Superior de Agricultura "Lniz de Queiroz", v.28, p.257-279, 1971 .

JOHNSON, C.M.; STOUT, P.R.; BROYER, T.C.; CARLTON, A.B. Comparative chlorine requirement of different plant species. Plant and Soil, v.8, n.3, p.337-353, 1957.

MALAVOLTA, E.; BOARETTO, A.E.; MURAOKA, T. Avaliaçũo do estado nutricional do arroz e fejjociro. Piracicaba: USP/CENA, 1993. 28P. (Apostila mimeografada).

MALAVOLTA, E.; VITTI, G.C.; OLIVEIRA, A.S. Avaliação do estado nutricional das plantas: princípios e aplicações. Piracicaba: Associação Brasileira para Pesquisa da Potassa e do Fosfato. 1989. 201p.

MALAVOLTA, E.; DAMIĀO FILHO, C.F.; VOLPE, C.A.; MACHADO JUNIOR, G.R.; VELHO, L.M.S.; ROSA, P.R.F.; LAURENTIZ, S. Deficiência e excessos minerais no feijoeiro (Phaseolus vulgaris L., cv. Carioca). Anais da Escola Superior de Agricultura “Luiz de Queiroz", v.37, n.2, p.701-718, 1980.

ULRICH, A. Plant analysis: methods and interpretation of results. In: KITCHEN, H.B. ed. Diagnostic techniques for soils and plants. Washington: American Potash Institute, 1948. p.157-198.

Entregue para publicação em 18.05.95

Aceito para publicação em 23.08.95 